University of Wollongong

Research Online

Faculty of Engineering and Information

Faculty of Engineering and Information

Sciences - Papers: Part A

Sciences

$1-1-2015$

Terahertz spectroscopy of 2,4-dinitrotoluene over a wide temperature range $(7-245 \mathrm{~K})$

Lucia Lepodise

University of Wollongong, Im1533@uowmail.edu.au

Josip Horvat

University of Wollongong, jhorvat@uow.edu.au

R A. Lewis

University of Wollongong, roger@uow.edu.au

Follow this and additional works at: https://ro.uow.edu.au/eispapers

Part of the Engineering Commons, and the Science and Technology Studies Commons

Research Online is the open access institutional repository for the University of Wollongong. For further information contact the UOW Library: research-pubs@uow.edu.au 


\title{
Terahertz spectroscopy of 2,4-dinitrotoluene over a wide temperature range
} $(7-245 \mathrm{~K})$

\author{
Abstract \\ Previous THz spectroscopy of the TNT explosive precursor, 2,4-dinitrotoluene (DNT), has been restricted \\ to room temperature (apart from one set of data at $11 \mathrm{~K}$ ). Here, for the first time, we investigate the \\ spectrum as the temperature is systematically varied, from 7 to $245 \mathrm{~K}$. Many new features appear in the \\ spectrum on cooling below room temperature. As well as the five absorption lines observed previously, \\ we observe five additional lines. In addition, a new room-temperature line at $8.52 \mathrm{THz}(281 \mathrm{~cm}-1)$ is \\ observed. Six of the lines red-shift with temperature and four of them blue-shift. The blue shift is \\ explained by interplay between intramolecular and intermolecular hydrogen bonds. The variation in line \\ width and line intensity with temperature is not systematic, although a conspicuous decrease in line \\ intensity with temperature is observed in all cases. Modeling with hybrid PBEO and TPSSh functionals \\ helps identify absorption modes.
}

\section{Keywords}

245,7 , range, temperature, wide, $k$, over, terahertz, dinitrotoluene, 4, 2, spectroscopy

\author{
Disciplines \\ Engineering | Science and Technology Studies
}

\section{Publication Details}

Lepodise, L. M.., Horvat, J. \& Lewis, R. A. (2015). Terahertz spectroscopy of 2,4-dinitrotoluene over a wide temperature range (7-245 K). The Journal of Physical Chemistry A: Isolated Molecules, Clusters,

Radicals, and Ions; Environmental Chemistry, Geochemistry, and Astrochemistry; Theory, 119 (2), 263-270.

This journal article is available at Research Online: https://ro.uow.edu.au/eispapers/3442 


\title{
Terahertz spectroscopy of 2, 4 dinitrotoluene over a wide temperature range $(7-245 \mathrm{~K})$
}

\author{
Lucia M. Lepodise, Joseph Horvat*, and R. A. Lewis \\ Institute for Superconducting and Electronic Materials and School of Physics, University of \\ Wollongong, Wollongong, New South Wales 2522, Australia
}

Previous THz spectroscopy of the TNT explosive precursor, 2, 4-dinitrotoluene (DNT), has been restricted to room temperature (apart from one set of data at $11 \mathrm{~K}$ ). Here, for the first time, we investigate the spectrum as the temperature is systematically varied - from $7 \mathrm{~K}$ to $245 \mathrm{~K}$. Many new features appear in the spectrum on cooling below room temperature. As well as the five absorption lines observed previously, we observe five additional lines. In addition, a new roomtemperature line at $8.52 \mathrm{THz}\left(281 \mathrm{~cm}^{-1}\right)$ is observed. Six of the lines red-shift with temperature and four of them blue-shift. The blue shift is explained by interplay between intra-molecular and inter-molecular hydrogen bonds. The variation in line width and line intensity with temperature is not systematic, although a conspicuous decrease in line intensity with temperature is observed in all cases. Modeling with hybrid PBE0 and TPSSh functionals helps identify absorption modes. 


\section{INTRODUCTION}

Many research groups ${ }^{1,2,-16}$ have measured the $\mathrm{THz}$ absorption spectra of explosives and related materials and they are found to have spectral fingerprints in the $\mathrm{THz}$ region. The spectral fingerprints are collectively due to the inter-molecular and intra-molecular vibrations. Various electromagnetic radiations such as $\mathrm{X}$ ray, microwave and gamma rays have been employed in explosive detection. The $\mathrm{THz}$ method has the advantages that it can penetrate a variety of materials including paper, clothing and ceramics while simultaneously only involving low photon energies and hence it does not cause any photo-ionization; moreover, it is a nondestructive technique.

The bulk of the previous work on THz spectroscopy of explosives has been performed at room temperature using THz-TDS. ${ }^{3,17}$ There are few reports on the THz spectra at low temperatures. Moreover, the available low-temperature reports focus on a particular temperature, without studying the temperature dependence of the spectrum over a broad range. For example, Laman et al. ${ }^{1}$ reported on 2, 4 dinitrotoluene (2, 4-DNT) cooled to $11 \mathrm{~K}$ while Ewelina et al. ${ }^{5}$ investigated various explosives cooled to $78 \mathrm{~K}$; in contrast, Burnett et al. ${ }^{18}$ studied RDX over a wide range of temperatures. In the present work, the temperature dependence of the THz spectrum of 2, 4-DNT is reported down to $7 \mathrm{~K}$. We observe several new low-temperature absorption lines below 100 $\mathrm{cm}^{-1}$, which are useful in studying structure and interactions in DNT. We also observe a new room-temperature absorption line at $8.52 \mathrm{THz}\left(281 \mathrm{~cm}^{-1}\right)$, which may be useful in security applications. In addition, for the first time, we provide detailed hybrid DFT calculations to identify the origin of the absorption lines. Both blue and red shift with temperature was obtained for low-energy absorption lines. This reveals the importance of the type of hydrogen bonding and associated charge transfer between the hydrogen-bonded molecules for the absorptions at energies below $100 \mathrm{~cm}^{-1}$. Our combined theoretical calculations and experimental study 
involving systematic variation of the temperature, provide new insights into the spectroscopy of 2, 4-DNT, and, by extension, other explosives and organic compounds.

\section{EXPERIMENTAL AND COMPUTATION METHODS}

The THz spectrum of a 2, 4-DNT pellet was measured using a FTIR Bomem DA8 spectrometer both at room temperature and at cryogenic temperatures. The 2, 4-DNT used was purchased from Sigma-Aldrich. Pellets of $1 \mathrm{~mm}$ thickness and $9 \mathrm{~mm}$ diameter were made by pressing a mixture of polyblend and 2, 4-DNT at 4500 psi for 30 seconds. The concentration of 2, 4-DNT in the pellet was $5 \%$ by weight. A continuous-flow Oxford Optistat cryostat was employed for cooling the sample. The cooling was through the use of helium gas. The sample was cooled from $245 \mathrm{~K}$ to $7 \mathrm{~K}$ with a step size of $14 \mathrm{~K}$. The spectra were collected with a resolution of $0.5 \mathrm{~cm}^{-1}(0.015 \mathrm{THz})$. More details of the experimental conditions can be found elsewhere ${ }^{19}$. Data analysis was via the IGOR software package. The absorption peaks obtained experimentally were fitted with Lorentzian curves to determine the line position, width, intensity and their corresponding uncertainties.

Numerical modeling of the absorption lines was performed with the ORCA software package ${ }^{20}$ on one and two molecules of 2, 4-DNT, in the harmonic approximation and without thermal excitations. The hybrid Perdew-Burke-Ernzerhoff gradient-corrected functional was used (PBE0), ${ }^{21,}{ }^{22}$ with $25 \%$ of Hartree-Fock exchange energy. Additionally, the Tao-PerdewStaroverov-Scuseria ${ }^{23}$ meta-generalized, gradient-corrected functional with $10 \%$ of Hartree-Fock exchange energy was used, TPSSh. ${ }^{24}$ The TPSSh method includes the kinetic energy density of the occupied orbitals and is an improvement over PBE0. For both, def2-TZVPP basis sets ${ }^{25} 26$ with Pople ++ diffuse functions ${ }^{27}$ and an empirical van der Waals correction (VDW06) ${ }^{28}$ were 
employed. Tight geometry optimization was performed before calculating the vibrational frequencies. No scaling of vibrational frequencies was used. For calculation of atomic charges with CHELPG method, ${ }^{29}$ def2-QZVPP basis sets with diffuse functions were used, coupled to TPSSh and SCS-MP2 $2^{30}$ theories corrected with VDW06.

\section{RESULTS AND DISCUSSION}

Figure 1 shows the optimized geometry of a single 2, 4-DNT molecule using TPSSh method. The ortho- and para- nitrous groups make dihedral angles of $28.5^{\circ}$ and $0.8^{\circ}$, respectively, with the benzene plane. The shortest distance between the nitrous oxygen and methyl hydrogen is $2.51 \AA$, implying an overlap of their van der Waals clouds and creation of intra-molecular hydrogen bond. The shortest distance between the aromatic hydrogen and nitrous oxygen is 2.41 $\AA$. The optimized geometry file is given in Supporting Information. The absorption lines obtained numerically for a single molecule correspond to the intra-molecular vibrations.

Figure 2 shows the experimental room temperature FTIR spectrum for 2, 4-DNT. The wavenumbers for the observed absorption lines are shown above the experimental spectrum. The position and relative intensities of the calculated infrared vibration modes using the PBE0 and TPSSh methods for single 2, 4-DNT molecule are shown by square and round symbols, respectively. There is a very good correspondence between the observed and calculated absorption lines at room temperature and thus the use of scaling factors ${ }^{33}$ was not necessary. All observed lines were accounted for by the modeling. There is a slight shift in energy between the PBE0 and TPSSh lines, with PBE0 giving a better agreement with experiment in terms of the line intensity. This may be due to higher proportion of the exact exchange energy in PBE0 than in TPSSh. Nevertheless, the agreement with experiment is remarkable, considering that the 
modeling was done on a single molecule. Crystal interactions are certain to affect the intensity and energy of the absorption lines. Good agreement between the non-scaled numerical and observed absorption energies shows that the anharmonic effects within DNT molecules are small. ${ }^{33}$ This agreement further indicates that the inter-molecular bonds are much weaker than the intra-molecular ones, affecting only weakly the intra-molecular vibrational energies.

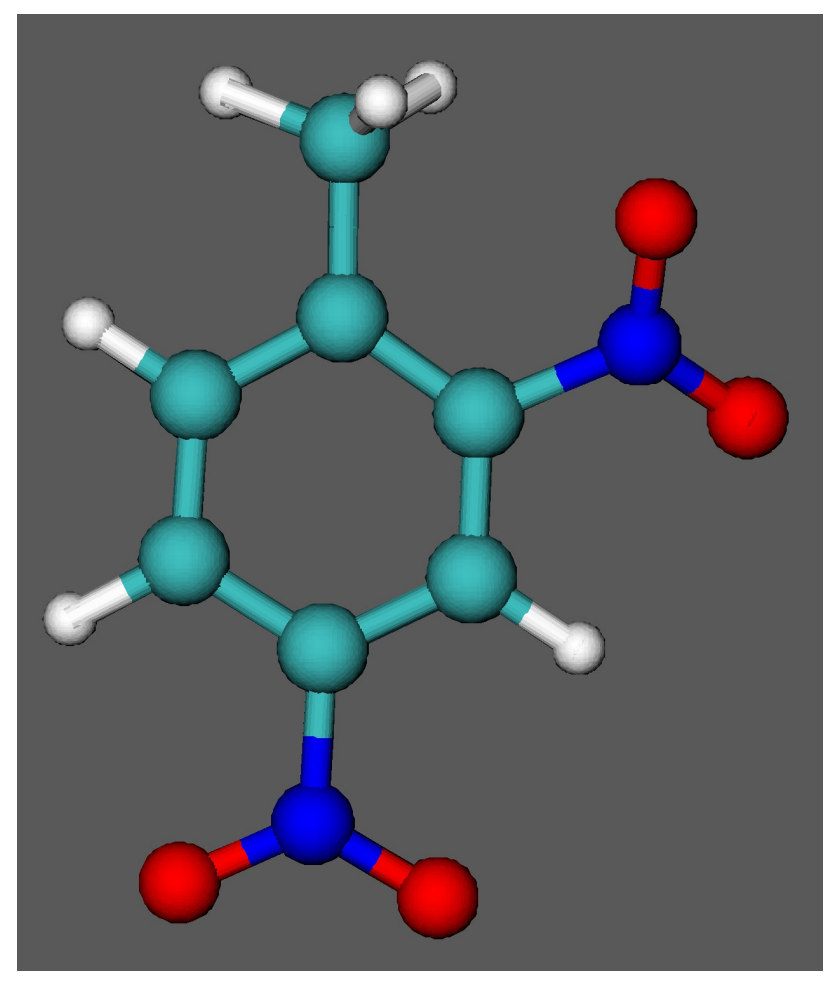

Figure 1. Calculated molecular structure of 2, 4-dinitrotoluene $\left(\mathrm{C}_{7} \mathrm{H}_{6} \mathrm{~N}_{2} \mathrm{O}_{4}\right)$ using the ORCA package with TPSSh method. Cyan spheres - C, red spheres - O, blue spheres - N, and white spheres - H. The molecular structure was plotted using VMD graphical software. ${ }^{32}$ 


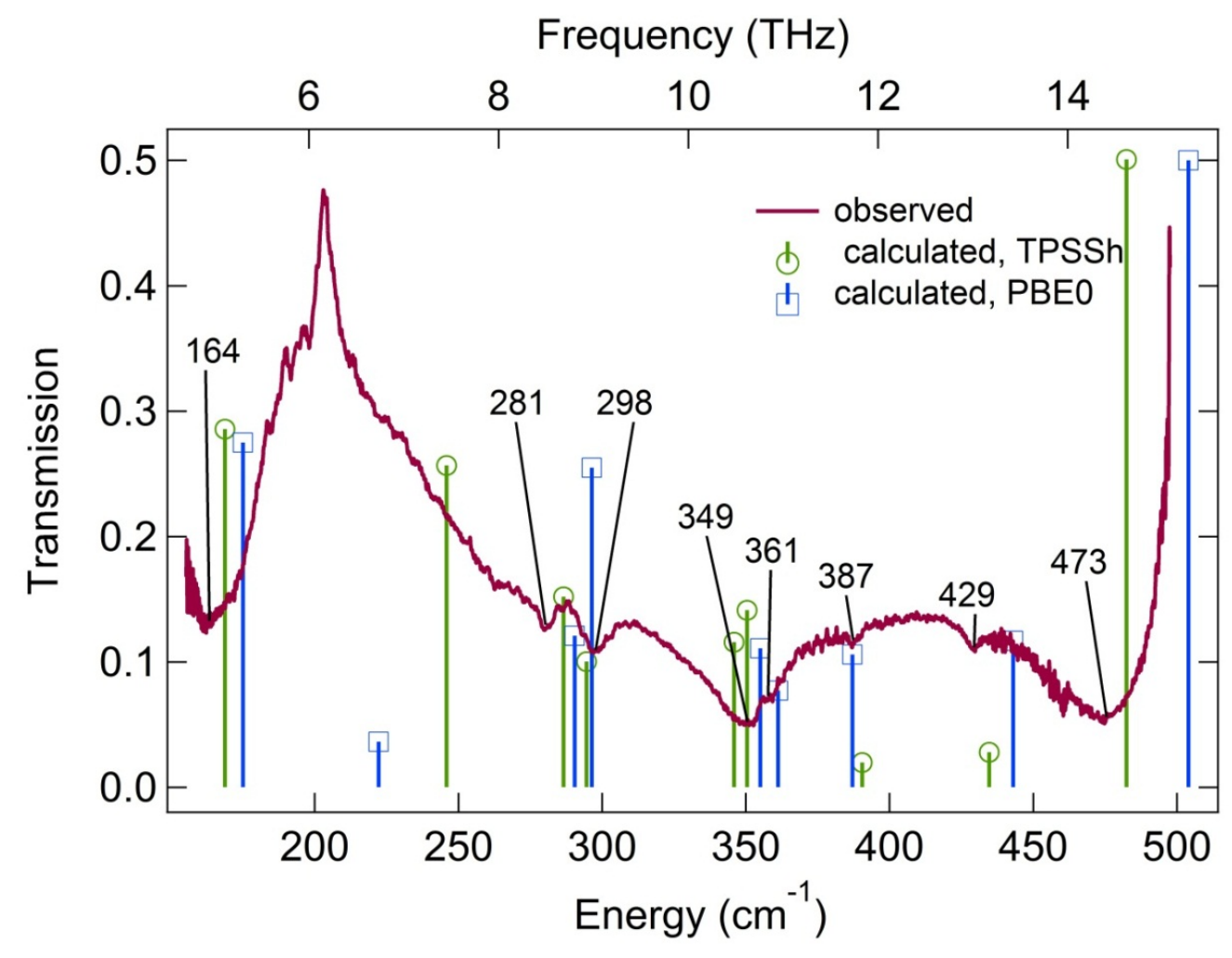

Figure 2. Room-temperature spectrum of 2, 4-dinitrotuluene in the high-frequency range, 5-15 THz, compared to the DFT numerical calculations obtained with two methods, PBE0 (squares) and TPSSh (circles). Positions of all experimental lines are given in $\mathrm{cm}^{-1}$.

Table 1 compares the absorption lines found in the literature by two independent groups and our measurements at room temperature, together with our calculated lines. There is a very good agreement between all these reports and our measurements. A notable difference is that we observe a distinct absorption line at $8.52 \mathrm{THz}=281 \mathrm{~cm}^{-1}$ (Fig. 2), which has not been reported by any of the other groups. We also resolved two absorption lines at 10.48 and $10.61 \mathrm{THz}$ (349 and $361 \mathrm{~cm}^{-1}$ ), whereas others report only one line near these energies. The PBE0 and TPSSh methods both reproduce the experimental line at $281 \mathrm{~cm}^{-1}$, as well as distinguishing the two lines at 349 and $361 \mathrm{~cm}^{-1}$ (Fig. 2). Both TPSSh and PBE0 methods also give a very good agreement 
with the remaining experimental lines. The difference in the energy of the room temperature lines obtained from the experiment and the TPSSh method is up to $12 \mathrm{~cm}^{-1}$, but typically less than $6 \mathrm{~cm}^{-1}$, which is noteworthy for a DFT-based method. Analysis of the calculated molecular vibrations using the TPSSh method associated with each of the absorption lines lead to the assignment of the intra-molecular absorption modes as shown in Table 1 and in the visualization multimedia files. The line calculated with TPSSh at $246 \mathrm{~cm}^{-1}\left(222 \mathrm{~cm}^{-1}\right.$ with PBE0) does not seem to have a corresponding experimental line. However, there is an experimental shoulder noticeable at these energies (Fig. 2). This calculated line corresponds to twist of $\mathrm{CH}_{3}$ group around its bond with benzene ring, counteracted by out-of-plane rocking of benzene ring (Media 2). However, we do not list it in Table 1, because experiment does not identify a distinct line. Two multimedia files were produced for some modes (with suffix $a$ or $b$ ), to help visualize both in-plane and out-of-plane vibrations. The mixing of the in-plane and out-of plane vibrations for these modes is most likely due to hydrogen and van der Waals and hydrogen bonds between the atoms in the molecule. For example, a pure in-plane vibration of the benzene ring would exert a pull on the out-of-plane atoms via the intra-molecular hydrogen and van der Waals interactions, mixing out-of-plane vibrations into a basically in-plane mode.

Having established the credentials of our methods at room temperature, we now turn to the temperature dependence of the absorption lines. None of the intra-molecular absorption lines in Table 1 changed significantly by cooling to $7 \mathrm{~K}$, within the used accuracy of our set-up. However, access to low temperatures enabled us to observe numerous additional absorption lines, which were obscured at room temperature by strong thermal excitations. These absorption lines correspond to inter-molecular vibrations and they do not appear in the numerical model of a single 2, 4-DNT molecule. 
Table 1. Comparison of experimental absorption lines with previous work at room temperature, as well as numerical results (TPSSh). The bold-face numbers are the literature values given in the original units. Associate multimedia files showing the monomer vibration modes are given as supporting information, to help visualize the vibration modes described in the table. Multimedia files were produced using VideoMach and $\mathrm{VMD}^{32}$ software.

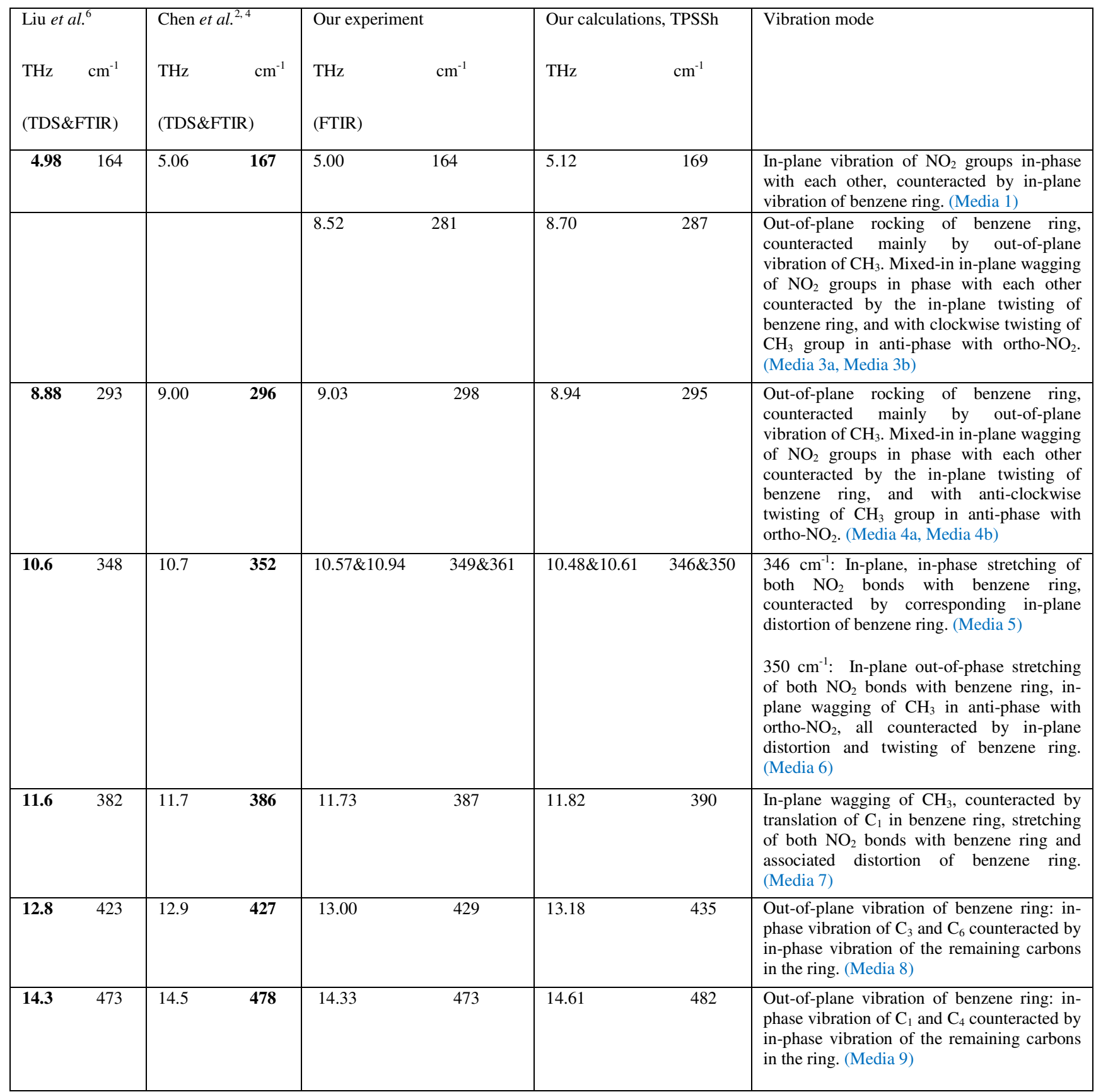


Figure 3 shows emergence of new absorption lines below $3 \mathrm{THz}$ at low temperatures. The strongest lines appear between 1.2 and $2 \mathrm{THz}$. Comparison with the literature (Table 2) shows that the absorption lines at 1.07, 1.24, 1.47, 1.66 and $1.96 \mathrm{THz}$ were also observed by Laman $e t$ $a l .{ }^{1}$, who used the Time-Domain Spectroscopy at $11 \mathrm{~K}$. Our FTIR measurements at both 7 and $21 \mathrm{~K}$ give new absorption lines at 1.14, 2.05, 2.11, 2.24 and $2.51 \mathrm{THz}$, in addition to the lines observed by Laman et al.. The absorption lines are in the far infrared region and hence they can be attributed to phonon or inter-molecular modes. This is a reasonable assumption, because a typical optical phonon frequency is $1-6 \mathrm{THz} .^{31}$ Figure 4 shows the result of the numerical calculations for two DNT molecules. Not all the experimental absorptions lines were obtained in the calculations because only two molecules were modeled. Calculations with more molecules could not be completed because they are excessively time consuming. However, it is unclear how reliable our line assignment is in this region, as many experimental lines were not accounted for. Figure 5 shows the optimized geometry of the two DNT molecules. Initial geometry for this dimer modeling was such that both molecules were in-plane and the out-of-plane structure shown in Fig. 5 was obtained after a lengthy geometry optimization using Tight Optimization option in Orca package, with convergence criterion for energy of $27 \times 10^{-6} \mathrm{eV}$. The optimized geometry was not exactly what is expected for this crystal. The dihedral angles between the aromatic plane and ortho- or para- nitro-groups were obtained as $20^{\circ}$ and $7^{\circ}$ respectively for one molecule, whereas they were both $15^{\circ}$ for the other molecule in our modeling. In the crystal, these values should span from $\sim 5^{\circ}$ to $\sim 45^{\circ}$ for different molecules in the unit cell. ${ }^{34}$ The two molecules were also tilted by $\sim 15^{\circ}$ in the modeling. Visualization files for obtained vibration modes are given as Supporting Information (Media 10 to 16). 
Frequency $(\mathrm{THz})$

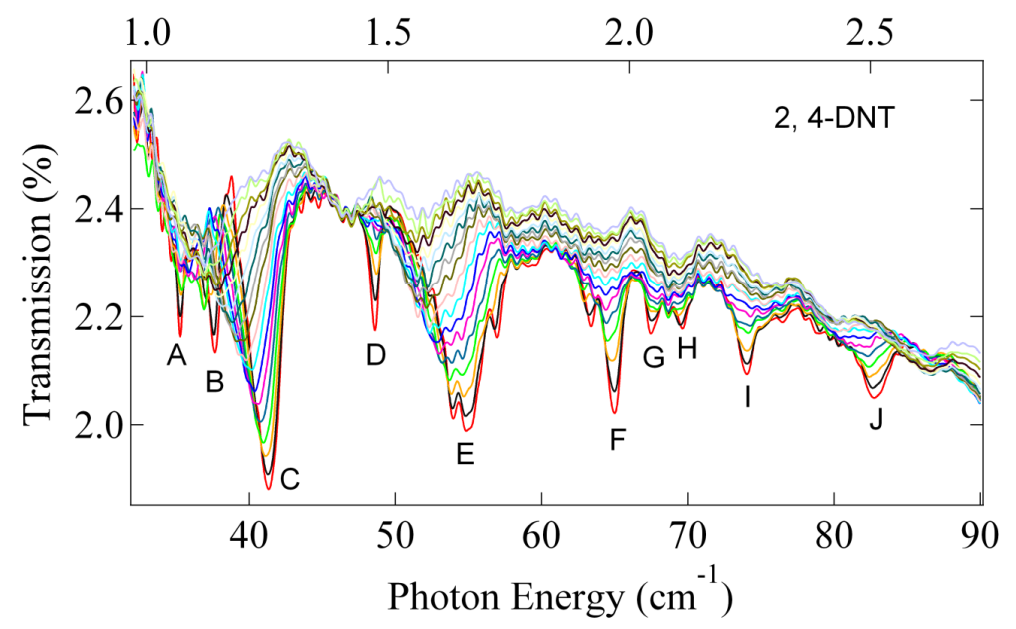

Figure 3. THz transmission spectra of 2, 4-dinitrotoluene at low temperatures. All the lowtemperature spectra have been offset along the vertical axis for clarity. Temperatures from top to bottom: 245, 231, 217, 203, 189, 175, 161, 147, 133, 119, 105, 91, 77, 63, 49, 35, 21 and $7 \mathrm{~K}$.

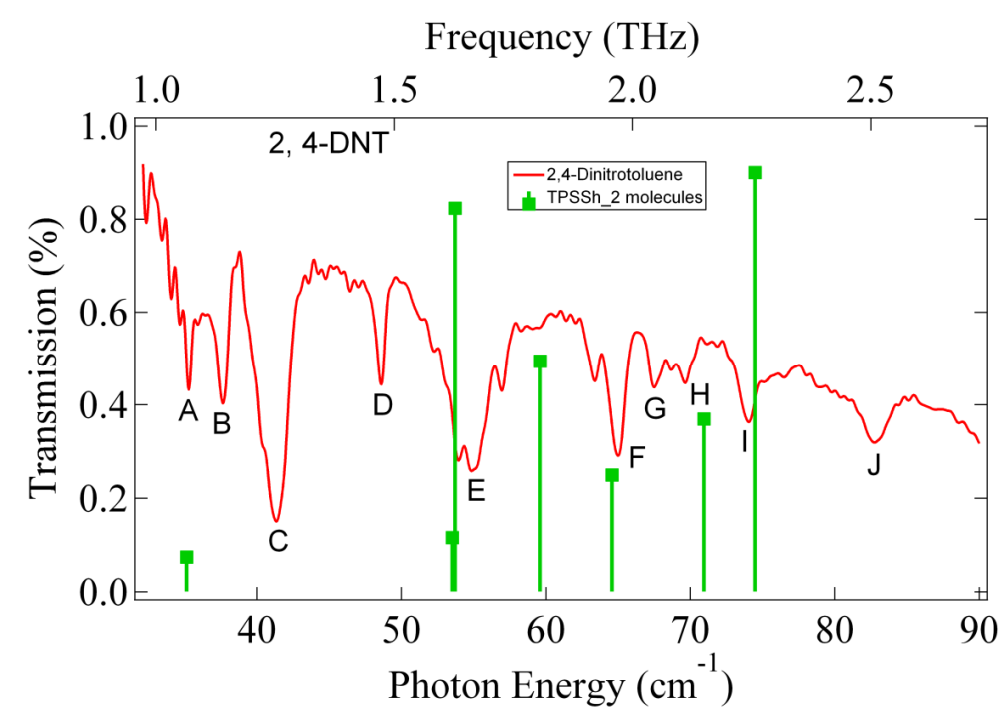

Figure 4. $\mathrm{THz}$ transmission spectrum of 2, 4-dinitrotoluene at $7 \mathrm{~K}$ compared to the numerical calculations using the TPSSh/def-2-QZVPP method for two molecules. 
Table 2. Comparison of observed absorption lines in the $32-90 \mathrm{~cm}^{-1}$ region with previous work, for both room temperature and low temperature measurements. The bold-face numbers are the literature values given in the original units.

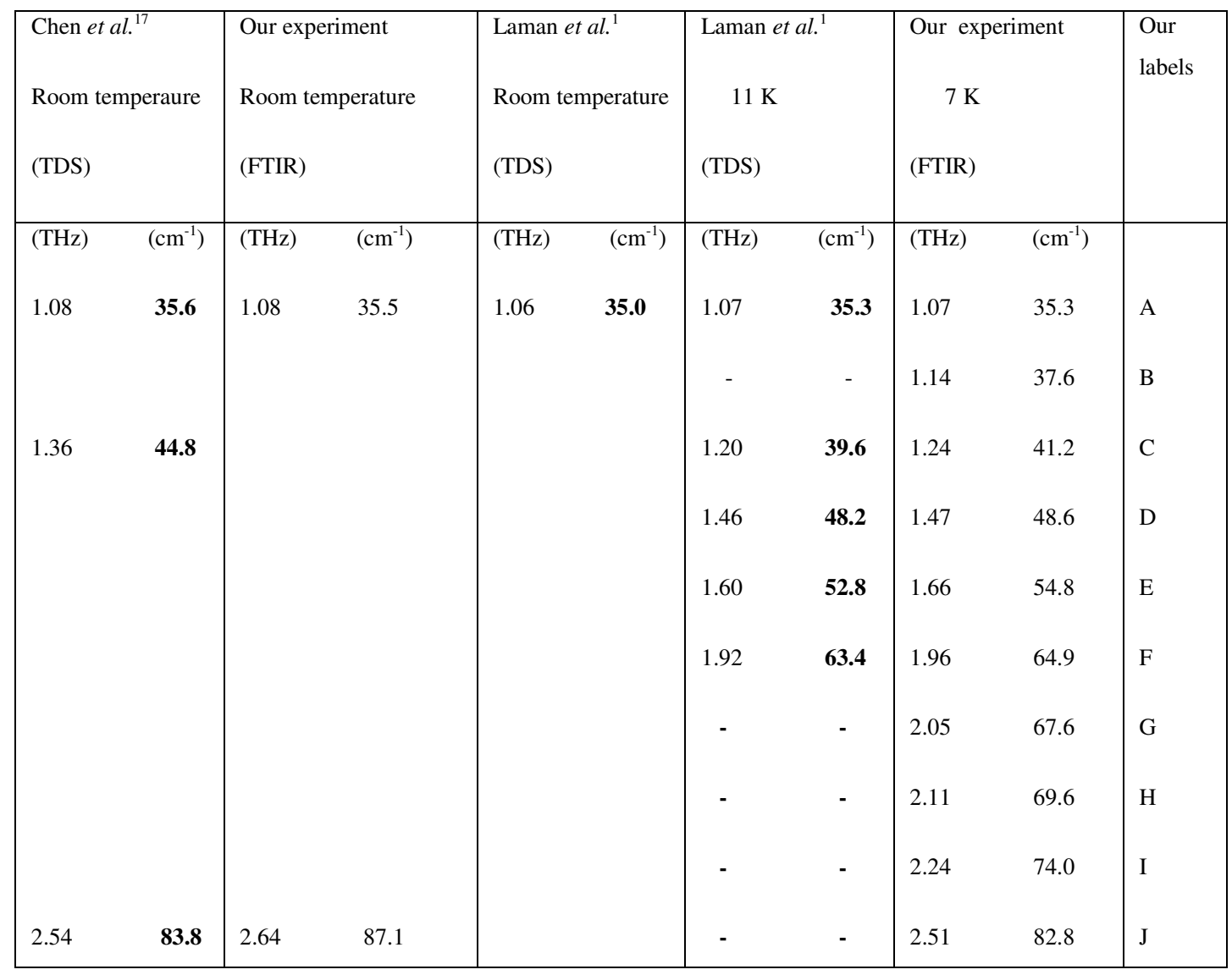




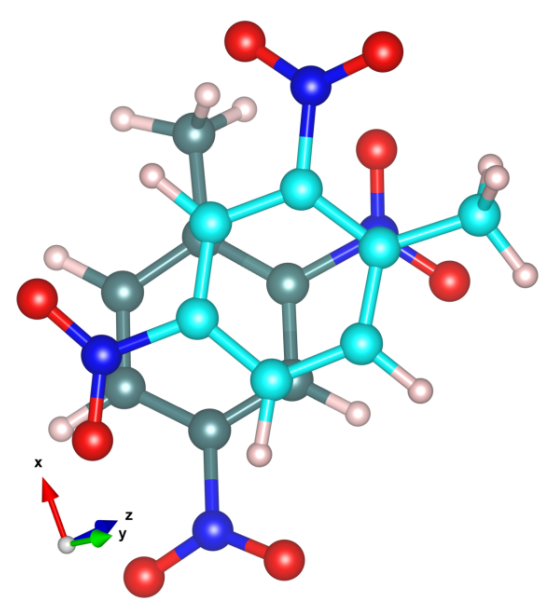

Figure 5. Optimized geometry of two 2,4-DNT molecules, using the TPSSh/def2-QZVPP method with ORCA package. Molecule 2 is on top of molecule 1. Carbon atoms for molecule 1 are shown as teal, while for the molecule 2 they are cyan, to distinguish the two more easy. Nitrogen, oxygen and hydrogen are blue, red and white, respectively. This image was produced using Vesta software.

Figure 6 shows the amount by which an absorption line shifts with temperature from its position at the lowest observed temperature $(7 \mathrm{~K})$. Most of the absorption lines red-shift with the temperature increase. This red shift appears to be linear in temperature. Such a shift is usually attributed to the increased bond lengths due to thermal expansion with warming ${ }^{36}$ and it is associated with the anharmonicity of the vibrational modes. ${ }^{1}$ However, the absorption lines A, D, $\mathrm{G}$ and I blue shift with temperature. All blue shifting lines are observed only below $100 \mathrm{~K}$. 


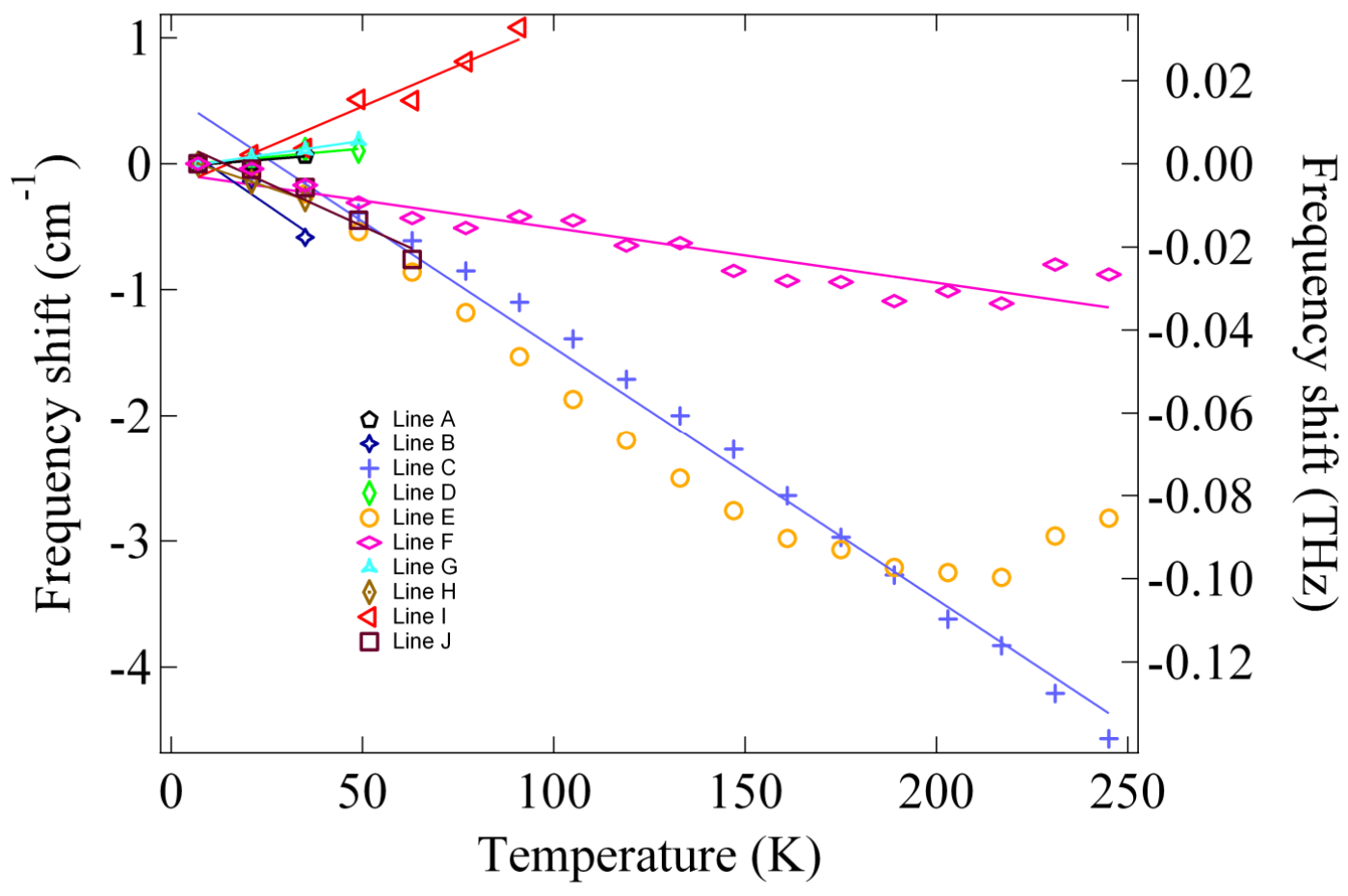

Figure 6. Change of the absorption line frequency from its initial position at the lowest temperature, plotted against temperature.

A distinct blue shift of absorption bands with temperature was also reported for sucrose by Walther et al.. ${ }^{41}$ Two absorption bands shift to higher energies with increasing temperature, reach a peak and then decrease in energy with further warming. Such blue shift was not reported for the absorption bands above $1.8 \mathrm{THz}$. A mechanism involving interplay between the hydrogen bonds and van der Waals bonds was suggested as the explanation for the observed peak in the blue shift. The blue shift for sucrose was shown to become more pronounced with deuteration. ${ }^{41}$ In the Born-Oppenheimer approximation, nuclear and electronic excitations are de-coupled, resulting in identical potential energy surfaces for hydrogen and deuterium. Consequently, the effect of replacing hydrogen with deuterium would simply be an increase of the vibrating 
molecule mass. This would be expected to lower the vibrational frequencies, contrary to the observed stronger blue shift with temperature after deuteration. While the Born-Oppenheimer approximation is applicable to most systems, it was recently shown that it cannot be used for treatment of hydrogen bonds. ${ }^{42}$ Rather, a numerical treatment that includes coupling of nuclear and electronic degrees of freedom is necessary to accurately describe the symmetry, energetics and isotope effect for a hydrogen bonded system, $[\mathrm{CNHNC}]$. It was shown that replacing hydrogen with a heavier isotope decreases the hydrogen bond length and that heavier isotope results in a larger electron density around the hydrogen site. ${ }^{42}$ Therefore, the isotope effect indicates the importance of hydrogen bonds on the blue shift with temperature in molecular crystals. Putting together the observed heightened vibrational blue shift with deuteration for sucrose $^{41}$ and the modelled electron density increase around H-site with deuteration for $[\mathrm{CNHNC}]^{-42}$ suggests that an increased electron density around $\mathrm{H}$-site is associated with the blue shift of hydrogen bond vibrational frequency.

Similar values of blue shift as for DNT, normalized to the vibrational frequency of the corresponding band, have also been reported for other crystalline carbohydrates. ${ }^{39}$ The blue shift was assigned to hydrogen bonds in molecular crystals, but no mechanism was presented. The red shift with very weak temperature dependence was assigned to van der Waals forces only. ${ }^{40}$

The distance between nitrous oxygens and methyl or aromatic hydrogens in each 2, 4 - DNT molecule in our modelling is $2.55 \AA$ or less. This is less than the sum of the van der Walls radii of hydrogen and oxygen and therefore hydrogen bonds can be formed between these atoms. The shortest inter-molecular distance between nitrous oxygen of one DNT molecule and methyl hydrogen of the other DNT molecule in the dimer obtained by our modelling is $2.34 \AA$. Therefore, these bonds qualify as intra-molecular hydrogen bonds. ${ }^{34}$ While hydrogen bonds are directional, highly bent inter-molecular hydrogen bonds have been reported. ${ }^{43}$ 
Thermal expansion of molecular crystals occurs through lengthening of the intermolecular bonds. These are van der Waals and hydrogen bonds and they are much weaker than the intramolecular covalent bonds. Therefore, the vibrational modes responsible for the blue shift with temperature should be affected by the thermal lengthening of the inter-molecular hydrogen bonds. While the calculated vibrational frequencies are not accurate enough to enable a clear identification of the observed absorption bands (Fig. 4), the corresponding vibrational modes should provide a fair idea of the molecular dynamics involved in this frequency range. In the following discussion we will show that the inter-molecular vibrational modes obtained by our modelling that involve the twist of ortho- $\mathrm{NO}_{2}$ (Media 12-16 in Supporting Information) can blueshift with temperature.

Nitrous oxygen forms an intra-molecular hydrogen bond with methyl hydrogen (between O-9 and $\mathrm{H}-17$ in Fig.7). Nitrous O-10 also forms an intra-molecular hydrogen bond with $\mathrm{H}-14$. The frequency of this $\mathrm{NO}_{2}$ twist around its bond with carbon ring (Fig.7) will be mainly determined by the hydrogen bonds between atoms $\mathrm{O}-9$ and $\mathrm{H}-17$, as well as between $\mathrm{O}-10$ and $\mathrm{H}-14$ for an isolated DNT molecule. Our modelling on single DNT molecule at TPSSh/def2-QZVPP level does give the ortho- $\mathrm{NO}_{2}$ twist mode at $55.9 \mathrm{~cm}^{-1}$ (Media 17). In the DNT dimer, $\mathrm{H}-17$ also forms an inter-molecular hydrogen bond with the nitrous oxygen of the second molecule (O-29 in Fig. 7). With formation of the inter-molecular hydrogen bond between $\mathrm{H}-17$ and O-29, a charge transfer is expected between all of the atoms contributing to these hydrogen bonds. ${ }^{37}$ This charge transfer will affect the frequency of the $\mathrm{NO}_{2}$ twist. Numerical modeling on DNT dimer at TPSSh/def2-QZVPP level gives the twist mode of the ortho- $\mathrm{NO}_{2}$ at $53.7 \mathrm{~cm}^{-1}$ (Media 12), at lower energy than for the single DNT molecule. This modelling was performed without taking into account thermal effects, effectively for very low temperatures. Thermal expansion of the inter-molecular hydrogen bond between $\mathrm{H}-17$ and O-29 will now affect this charge transfer and 
twist frequency of the ortho- $\mathrm{NO}_{2}$ group. Because the ortho- $\mathrm{NO}_{2}$ twist mode undergoes a red shift with formation of DNT dimer, thermal lengthening of the bond between $\mathrm{H}-17$ and O-29 should result in a blue shift of the ortho- $\mathrm{NO}_{2}$ twist mode. Intra-molecular geometry will undergo much less thermal change, because much stronger covalent bonds are involved. This is our proposed mechanism for the observed blue shift with temperature. In the dimer, there are several modes involving ortho- $\mathrm{NO}_{2}$ twist, as inter- and intra- molecular modes are coupled. This would account for more than one observed band blue-shifting with temperature (Fig. 6). On the other hand, this mode-coupling could also result in the shift of the vibrational energies by itself. The accuracy of the vibrational energy calculus is not great, even though the numerical errors are cancelled out when the difference between two modes is calculated. Therefore, comparison of vibrational energies obtained with the same method should be more reliable. Nevertheless, further verification of the proposed blue-shift mechanism is desirable.

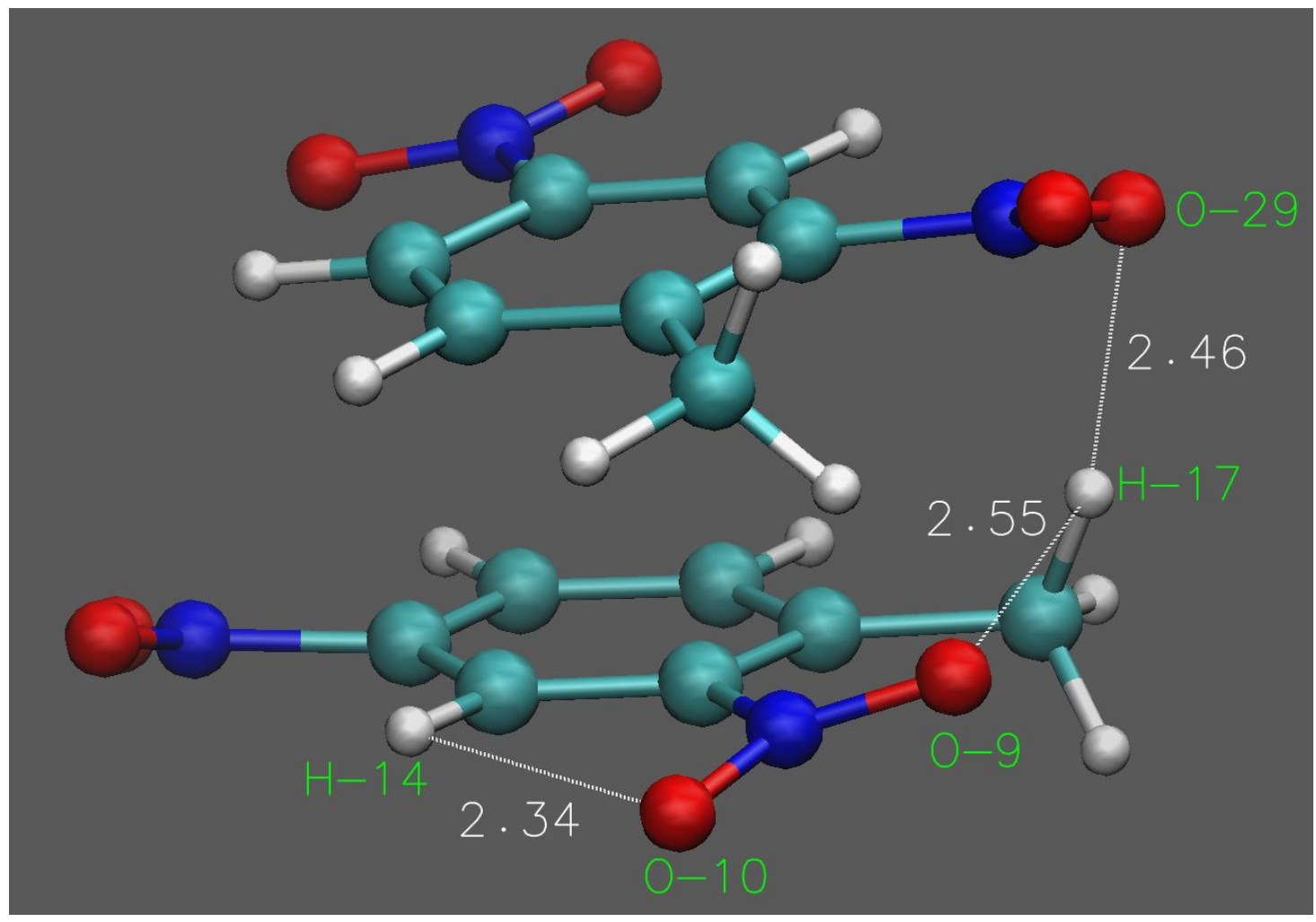


Figure 7: Geometry of DNT dimer, showing the hydrogen bonds (broken lines) relevant to twist vibrations of ortho- $\mathrm{NO}_{2}$ group. The geometry optimization was performed at TPSSh/def2QZVPP level. The atoms are carbon (teal), hydrogen (white), oxygen (red) and nitrogen (blue). Length of hydrogen bonds is given in $\AA$ (white). Select atoms are labeled in green (H-14 etc.). This figure was created using VMD graphical software. ${ }^{32}$

We further study how the formation of the dimer affects the charge distribution on O-9, O-10, H-14 and H-17 (Fig. 7). This can provide information on the associated vibrational energy change for hydrogen-bonded atoms in the molecule. ${ }^{38,41,42}$ The thermal expansion is then accounted for by moving the second DNT molecule away from its numerically optimized position along the hydrogen bond between $\mathrm{H}-17$ and O-29, by 5\% of this bond length. Ensuing charge distribution then shows whether the vibrational modes of ortho- $\mathrm{NO}_{2}$ undergo blue shift with temperature, as implied through comparison of the experiment and modelling of deuterated hydrogen bonds. ${ }^{41,42}$ Atomic charges in the molecules were calculated using CHELPG scheme. ${ }^{29}$ The atomic charges in this scheme are fitted to reproduce the electrostatic potential at grid points around the molecule. This is a more reliable method than the standard charge population schemes (Mulliken, Löwdin) commonly found in the quantum chemistry computational packages, providing large enough grid density is used. We used the grid spacing of $0.15 \AA$, half of the default value in ORCA. The atoms of interest are at the edges of DNT dimer, where CHELPG method is accurate. To ensure that our atomic charge re-distribution is not an artefact of the DFT-based method used (TPSSh), CHELPG analysis was performed at TPPSSh/def2QZVPP and also at RI-SCS-MP2/def2-QZVPP level. RI-SCS-MP2 is the Spin Component Scaled MP2 method, ${ }^{30}$ with Resolution of Identity approximation. SCS-MP2 is substantially more reliable than the plain $\mathrm{MP} 2^{30}$ and they are both Hartree-Fock-based methods. 
CHELPG analysis shows that the charges of atoms $\mathrm{O}-9, \mathrm{O}-10$ and $\mathrm{H}-17$ become more positive with the formation of the dimer (Table 3). Therefore, electron depletion occurs for these atoms. Charge of $\mathrm{H}-14$ becomes more negative, but changing by less than half of the amount occurring for the other 3 atoms. Qualitatively the same was obtained with TPSSh and SCS-MP2 methods. This implies that the twist frequency of ortho- $\mathrm{NO}_{2}$, containing the hydrogen-bonding O-9 and O10, decreases with the formation of the dimer. ${ }^{41,42}$ This is in agreement with our calculated change of the ortho- $\mathrm{NO}_{2}$ twist frequency, which decreased from $55.9 \mathrm{~cm}^{-1}$ to $53.7 \mathrm{~cm}^{-1}$ with the formation of the DNT dimer.

Table 3: Atomic charges (in multiples of electron charge, $e$ ) obtained in CHELPG analysis for optimized geometries of single DNT molecule (Q(DNT1)), DNT dimer (Q(DNT2)) and for the dimer where one molecule was shifted away from the other by $5 \%$ of the bond length between H-17 and O-29 (Q(DNT2_5\%)). The charges are given for both TPSSh and Spin Component Scaled-MP2 method.

\begin{tabular}{|c|c|c|c|c|c|c|c|c|c|c|}
\hline \multirow{2}{*}{$\begin{array}{l}\text { Atom } \\
\text { (Fig. 7) }\end{array}$} & \multicolumn{2}{|c|}{$\begin{array}{c}\mathrm{Q}(\mathrm{DNT} 1) \\
(e) \\
\end{array}$} & \multicolumn{2}{|c|}{$\begin{array}{c}\mathrm{Q}(\mathrm{DNT} 2) \\
(e) \\
\end{array}$} & \multicolumn{2}{|c|}{$\begin{array}{c}\mathrm{Q}\left(\mathrm{DNT} 2 \_5 \%\right) \\
(e) \\
\end{array}$} & \multicolumn{2}{|c|}{$\begin{array}{c}\mathrm{Q}(\mathrm{DNT} 2)-\mathrm{Q}(\mathrm{DNT} 1) \\
(e)\end{array}$} & \multicolumn{2}{|c|}{$\begin{array}{c}\mathrm{Q}\left(\mathrm{DNT} 2 \_5 \%\right)-\mathrm{Q}(\mathrm{DNT} 2) \\
(e)\end{array}$} \\
\hline & TPSSh & SCS-MP2 & TPSSh & SCS-MP2 & TPSSh & SCS-MP2 & TPSSh & SCS-MP2 & TPSSh & SCS-MP2 \\
\hline O-9 & -0.4053 & -0.4482 & -0.3766 & -0.4278 & -0.3784 & -0.4309 & 0.0287 & 0.0204 & -0.0018 & -0.0031 \\
\hline O-10 & -0.4024 & -0.4468 & -0.3857 & -0.4340 & -0.3876 & -0.4373 & 0.0167 & 0.0128 & -0.0019 & -0.0033 \\
\hline H-14 & 0.2250 & 0.2494 & 0.2189 & 0.2471 & 0.2196 & 0.2461 & -0.0061 & -0.0023 & 0.0007 & -0.0010 \\
\hline $\mathrm{H}-17$ & 0.1888 & 0.1922 & 0.2406 & 0.2656 & 0.2347 & 0.2573 & 0.0517 & 0.0734 & -0.0058 & -0.0083 \\
\hline
\end{tabular}


The effect of thermal expansion on the atomic charge distribution can be seen through CHELP analysis of the DNT dimer, for which the bond between H-17 and O-29 was lengthened by 5\% along this bond (Table 3). We chose relatively large 5\% to ensure that the bond lengthening effect is larger than the uncertainty of the numerical procedure. The atomic charges become more negative with the $5 \%$ bond lengthening when using TPSSh method, by $0.5 \%, 0.5 \%$ and $2.4 \%$ for $\mathrm{O}-9, \mathrm{O}-10$ and $\mathrm{H}-17$, respectively. The atomic charge of $\mathrm{H}-14$ becomes more positive by $0.3 \%$, which is a substantially smaller change than for the other atoms. Using SCS-MP2 method, the atomic charges of all 4 atoms become more negative with the lengthening of the inter-molecular hydrogen bond, by $0.7 \%, 0.7 \%, 0.4 \%$ and $3.1 \%$ for $\mathrm{O}-9, \mathrm{O}-10, \mathrm{H}-14$ and $\mathrm{H}-17$, respectively. This shows that the intra-molecular hydrogen bonds of ortho- $\mathrm{NO}_{2}$ group become electron-enriched with thermal expansion. As a result, the ortho- $\mathrm{NO}_{2}$ twist mode shifts to higher energies. Therefore, atomic charge distribution analysis confirms the viability of the model we propose for the observed blue shift with temperature (Fig. 6).

Another distinctive feature of the absorption lines is the reduction in the line width with decreasing temperature as well as the sharpening of the line, as shown in Fig. 8 and Fig. 9, respectively. This behavior is systematic for all absorption lines at temperatures below $100 \mathrm{~K}$, while there are fluctuations in both the line width and the line intensity for the lines $\mathrm{C}$ and $\mathrm{E}$ at higher temperatures. Interestingly, the frequency shift of the line $\mathrm{C}$ is linear throughout the temperature range, while it is anomalous for the line $\mathrm{E}$ above $200 \mathrm{~K}$. 


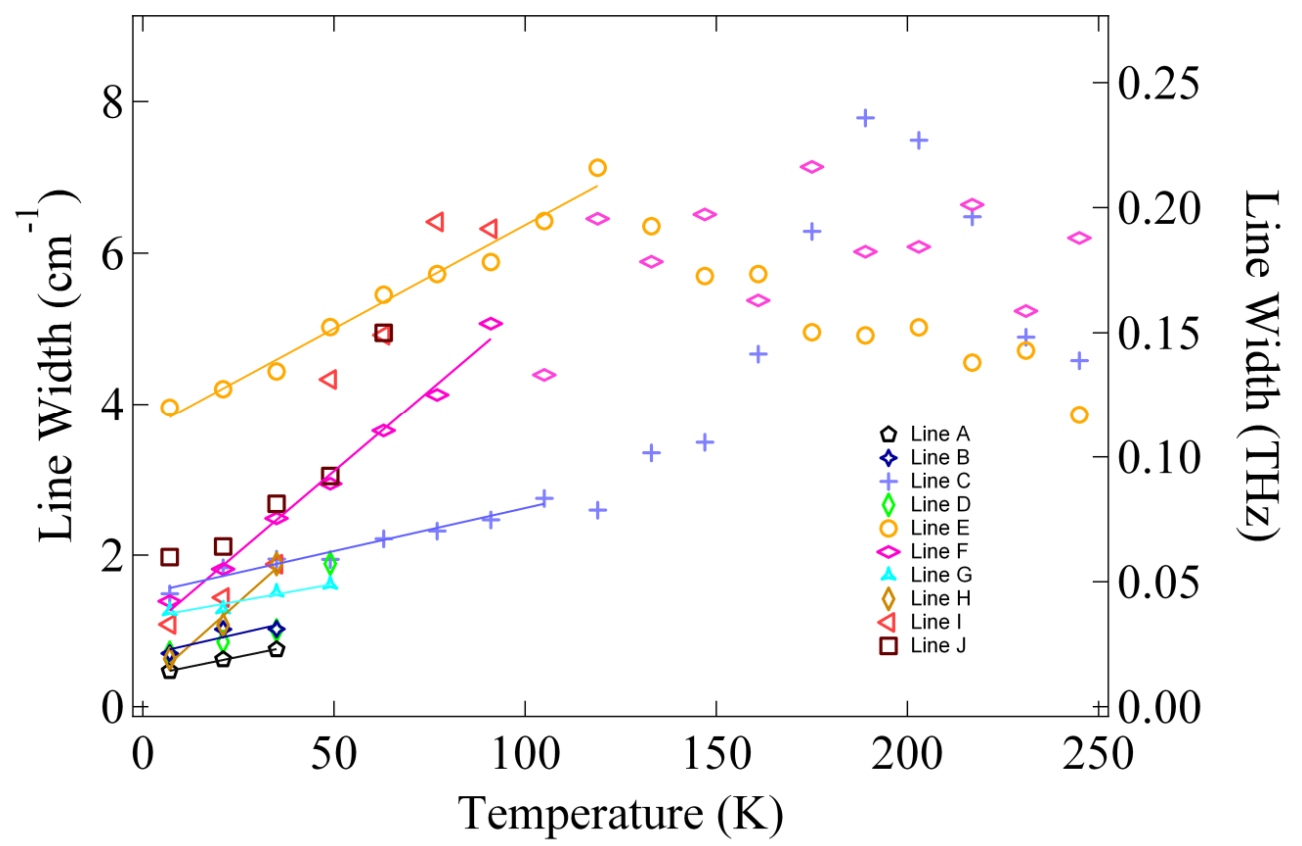

Figure 8. Change of the absorption line width with temperature.

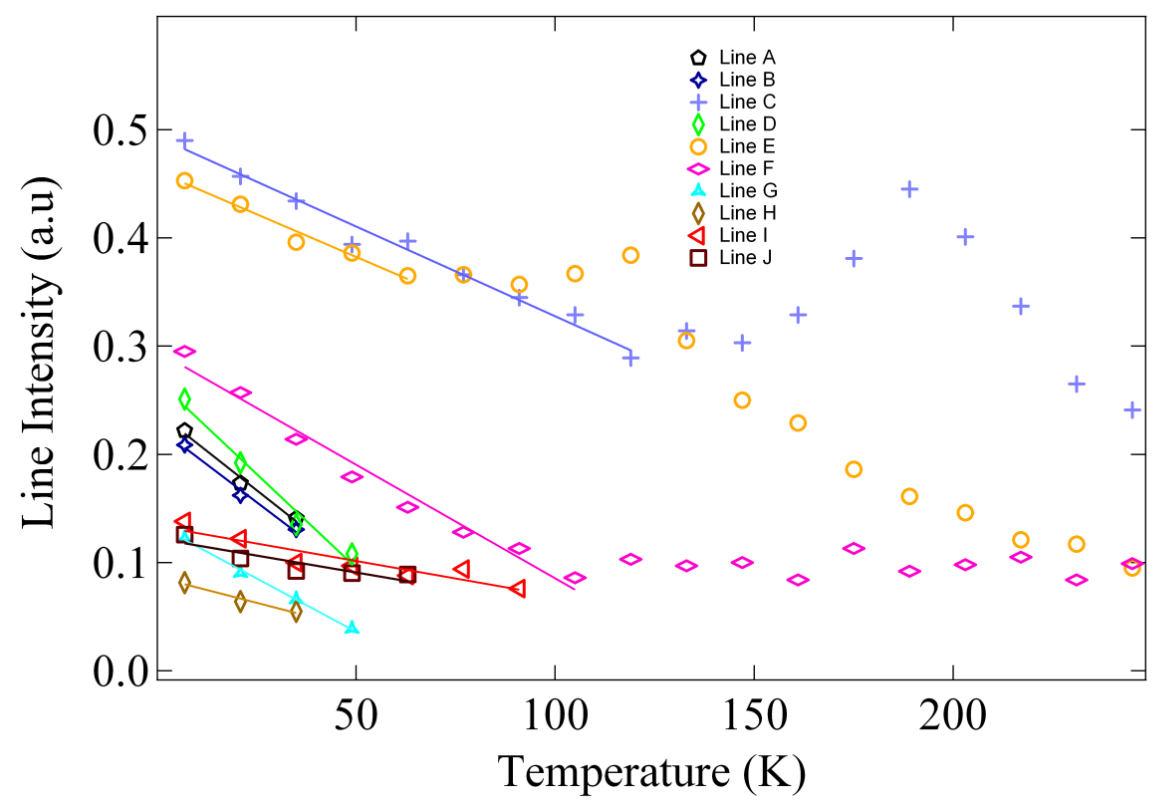

Figure 9. Change in absorption line intensity with temperature. 
The rates at which the energy, the line width and line intensity changed with temperature are presented in Table 4. The parameters in this table were extracted from the observed spectra assuming a linear temperature dependence of the three properties of the lines. It was observed that most of the red-shifting lines shift at higher rates than the blue-shifting lines (Table 4). Interestingly, the ratio of the intensity decrease rate to the energy shift rate is almost the same (close to 0.2 a.u. $/ \mathrm{cm}^{-1}$ ) for six out of ten lines (not the lines A, B, C and F). There was no straightforward trend observed in the rate of line width change. In some instances it is high for lines which blue shift with temperature and other instances it is high for lines which red shift with temperature.

Table 4. Change with temperature of the main properties of the absorption lines.

\begin{tabular}{|l|cc|cc|l|}
\hline Line Label & \multicolumn{2}{|c|}{ Position shift } & \multicolumn{2}{c|}{ Line width shift } & Intensity decrease \\
& $\left(\mathrm{cm}^{-1} / \mathrm{K}\right)$ & $(\mathrm{MHz} / \mathrm{K})$ & $\left(\mathrm{cm}^{-1} / \mathrm{K}\right)$ & $(\mathrm{MHz} / \mathrm{K})$ & $($ a.u./K $)$ \\
\hline A & 0.0024 & 72 & 0.0102 & 306 & 0.0017 \\
C & -0.0209 & -626 & 0.0116 & 348 & 0.0016 \\
D & -0.0200 & -600 & 0.0114 & 342 & 0.0021 \\
E & 0.0039 & 117 & 0.0262 & 786 & 0.0010 \\
F & -0.0181 & -543 & 0.0255 & 765 & 0.0035 \\
G & -0.0079 & -237 & 0.0429 & 1290 & 0.0003 \\
H & 0.0044 & 132 & 0.0091 & 273 & 0.0009 \\
I & -0.010 & -300 & 0.0045 & 135 & 0.0020 \\
\hline
\end{tabular}




\begin{tabular}{|l|ll|ll|l|}
\hline $\mathrm{J}$ & -0.0137 & -411 & 0.0492 & 1480 & 0.0029 \\
\hline
\end{tabular}

\section{CONCLUSION}

Intra-molecular absorption lines of 2,4-DNT are observed above $2.6 \mathrm{THz}$ and they do not change with cooling the sample to $7 \mathrm{~K}$. These lines were reproduced accurately in numerical modeling of single 2,4-DNT molecule at the PBE0/def2-TZVPP and TPSSh/def2-TZVPP level. We observed a new room-temperature intra-molecular line at $8.52 \mathrm{THz}$, which corresponds to out-of-plane rocking of benzene ring, counteracted by out-of-plane vibration of $\mathrm{CH}_{3}$. Intermolecular absorption lines start appearing below $250 \mathrm{~K}$ and they shift with temperature. Modeling with two DNT molecules gives 7 lines, however it is difficult to identify which experimental lines they correspond to. Our measurements give five absorption lines at $7 \mathrm{~K}$ below 2.6 THz that have not been reported before. The line width of all these absorption lines increases with temperature below $100 \mathrm{~K}$, while for the two strongest lines it decreases with temperature above 100 K. Similarly, the line intensity decreases with temperature for all lines below $100 \mathrm{~K}$, while for the two strongest lines the intensity attains a maximum between 100 and $200 \mathrm{~K}$. Most of the absorption lines below $2.6 \mathrm{THz}$ red shift linearly with increase of temperature. However, four of the inter-molecular lines blue shift with temperature. The blue shift was explained through interaction between intra- and inter-molecular hydrogen bonds. The ortho- $\mathrm{NO}_{2}$ group forms intra-molecular hydrogen bonds with methyl and aromatic hydrogen. However, the same methyl hydrogen also forms an inter-molecular hydrogen bond with $\mathrm{NO}_{2}$ group of a neighboring DNT molecule. Creation of DNT dimer through this inter-molecular hydrogen bond results in electron depletion in the intra-molecular hydrogen bonds. This results in red-shift of ortho- $\mathrm{NO}_{2}$ twist mode. Thermal expansion of the inter-molecular hydrogen bond partially restores electron 
density of intra-molecular hydrogen bonds, resulting in blue shift with temperature of the ortho$\mathrm{NO}_{2}$ twist mode. Mixing of the ortho- $\mathrm{NO}_{2}$ twist mode through inter-molecular interactions produces more than one blue-shifting inter-molecular mode.

\section{AUTHOR INFORMATION}

\section{Corresponding author}

*Email: jhorvat@uow.edu.au, Phone: 61-2-4221 8073

The authors declare no competing financial interests.

\section{ACKNOWLEDGEMENTS}

This work was supported by the Australian Research Council, the University of Wollongong, and the Botswana International University of Technology. Numerical modeling was performed at University of Wollongong High Performance Computing Centre.

Supporting Information Available: Atomic coordinates optimized by TPSSh method are given in .xyz format for single DNT molecule and dimer. Visualization files showing the 
vibration modes are available in .mpeg format. Tentative assignment of the vibrational modes based on dimer modelling is given. 


\section{REFERENCES}

(1) Laman, N.; Harsha, S. S.; Grischkowsky, D.; Melinger, J. S. High-resolution waveguide THz spectroscopy of biological molecules. Opt. Express. 2008, 16, 4094-4105.

(2) Chen, Y.; Liu, H.; Deng, Y.; Veksler, D. B.; Shur, M. S.; Zhang, X.-C.; Schauki, D.; Fitch, M. J.; Osiander, R.; C. Dodson, C.; Spicer, J. B. Spectroscopic characterization of explosives in the far-infrared region. Proc. SPIE 5411, Terahertz for Military and Security Applications II, 1 (September 8, 2004); doi:10.1117/12.540945.

(3) Gordon, B. M. Developments of THz imaging systems for detect of chemicals. CenSSIS Annual reports. Paper 13 (2008).

(4) Chen, Y.; Liu, H.; Deng, Y.; Schauki, D.; Fitch, M. J.; Osiander, R.; Dodson, C.; Spicer, J. B.; Shur, M.; Zhang, X.-C. THz spectroscopic investigations of 2, 4-dinitrotoluene. Chem. Phys. Lett. 2004, 400, 357-361.

(5) Witko, E. M.; Korter, T. M.; Wilkinson, J.; Ouellette, W.; Lightstone, J. Terahertz spectroscopy of energetic materials. Proc. SPIE 8023, Terahertz Physics, Devices, and Systems V: Advance Applications in Industry and Defense, 80230M (May 25, 2011); doi:10.1117/12.882905.

(6) Liu, H.-B.; Zhong, H.; Karpowicz, N.; Chen, Y.; Zhang, X.-C. Terahertz spectroscopy and imaging for defense and security applications. Proc. IEEE. 2007, 95, 1514-1527.

(7) Hu, Y.; Huang, P.; Guo, L.; Wang, X.; Zhang, C. Terahertz spectroscopic investigations of explosives. Phys. Lett. A. 2006, 359, 728-732. 
(8) Lo, T.; Gregory, I. S.; Baker, C.; Taday, P. F.; Tribe, W. R.; Kemp, M. C. The very farinfrared spectra of energetic materials and possible confusion materials using terahertz pulsed spectroscopy. Vibr. Spectr. 2006, 42, 243-248.

(9) Leahy-Hoppa, M. R.; Fitch, M. J.; Zheng, X.; Hayden, L. M.; Osiander, R. Wideband terahertz spectroscopy of explosives. Chem. Phys. Lett. 2007, 434, 227-230.

(10) Guo, L.; Zhang, L.; Wang, X.; Hu, Y.; Zhang, C. Time-resolved terahertz spectroscopy of explosive material. Chin. Opt. Lett. 2005, 3, S117-S119.

(11) Witko, E. M.; Korter, T. M. Terahertz Spectroscopy of the Explosive Taggant 2, 3Dimethyl-2, 3-Dinitrobutane. J. Phys. Chem A. 2012, 116, 6879-6884.

(12) Kemp, M. C. Explosives Detection by Terahertz Spectroscopy-A Bridge Too Far?

IEEE Transactions on Terahertz Science and Technology. 2011, 1, 282-292.

(13) Shen, Y. C.; Lo, T.; Taday, P. F.; Cole, B. E.; Tribe, W. R.; Kemp, M. C. Detection and identification of explosives using terahertz pulsed spectroscopic imaging. Appl. Phys. Lett. 2005, 86, 241116.

(14) Barber, J.; Hooks, D. E.; D. J. Funk, D. J. Temperature-Dependent Far-Infrared Spectra of Single Crystals of High Explosives Using Terahertz Time-Domain Spectroscopy. J. Phys. Chem. A. 2005, 109, 3501-3505.

(15) Kong, S. G.; Wu, D. H. Terahertz Time-Domain Spectroscopy for Explosive Trace Detection. Proceedings of the 2006 IEEE International Conference on Computational Intelligence for Homeland Security and Personal Safety. 2006, 47-50. 
(16) Fan, W. H.; Burnett, A.; Upadhya, P. C.; Cunningham, J.; Linfield, E. H.; Davies, A. G. Far-Infrared Spectroscopic Characterization of Explosives for Security Applications Using Broadband Terahertz Time-Domain Spectroscopy. Appl. Spectr. 2007, 61, 638-643.

(17) Chen, J.; Chen, Y.; Zhao, H.; Bastiaans, G. J.; Zhang, X.-C. Absorption coefficients of selected explosives and related compounds in the range of 0.1-2.8 THz. Opt. Express. 2007, 15, 12060-12067.

(18) Burnett, A. D.; Fan, W. H.; Upadhya, P. C.; Cunningham, J. E.; Edwards, H. G. M.; Kendrick, J.; Munshi, T.; Hargreaves, M.; Linfield, E. H.; Davies, A. G. Broadband terahertz time-domain and Raman spectroscopy of explosives. Proc. SPIE 6549, Terahertz for Military and Security Applications V, 654905 (May 04, 2007); doi:10.1117/12.719283 (2007).

(19) Lepodise, L. M.; Horvat, J.; Lewis, R. A. Collective librations of water molecules in the crystal lattice of rubidium bromide: experiment and simulation. Phys. Chem. Chem. Phys. 2013, 15, 20252-20261.

(20) Neese, F. The ORCA program system. WIREs-Comput. Mol. Sci. 2012, 2, 73-78.

(21) Perdew, J. P.; Burke, K.; Ernzerhof, M. Generalized gradient approximation made simple.

Phys. Rev. Lett. 1996, 77, 3865.

(22) Burke, K. Perspective on density functional theory. J. Chem. Phys. 2012, 136, 150901.

(23) Tao, J.; Perdew, J. P.; Staroverov, V. N.; Scuseria, G. E. Climbing the density functional ladder: nonempirical meta-generalized gradient approximation designed for molecules and solids. Phys. Rev. Lett. 2003, 91, 146401. 
(24) Staroverov, V. N.; Scuseria, G. E.; Tao, J.; Perdew, J. P. Comparative assessment of a new non empirical density functional: Molecules and hydrogen-bonded complexes. J. Chem. Phys. 2003, 119, 12129.

(25) Schaffer, A.; Horn, H.; Ahlrichs, R. J. Fully optimized contracted Gaussian basis sets for atoms Li to Kr. Chem. Phys. 1992, 97, 2571.

(26) Weigend, F.; Ahlrichs, R. Balanced basis sets of split valence, triple zeta valence and quadruple zeta valence quality for $\mathrm{H}$ to $\mathrm{Rn}$ : Design and assessment of accuracy. Phys. Chem. Chem. Phys. 2005, 7, 3297-3305.

(27) Krishnan, R.; Brinkley, J. S.; Seeger, R.; Pople, J. A. Self-consistent molecular orbital methods. XX. A basis set for correlated wave functions. J. Chem. Phys. 1980, 72, 650.

(28) Grimme, S. Comput. Chem. 2006, 27, 1787-1799.

(29) Breneman, C. M. and Wiberg, K. B. Determining atom-centered monopoles from molecular electrostatic potentials. The need for high sampling density in formamide conformational analysis. J. Comput. Chem.1990, 11, 361-373.

(30) Grimme, S. Improved second-order Møller-Plesset perturbation theory by separate scaling of parallel- and antiparallel-spin pair correlation energies. J. Chem. Phys. 2003, 118, 9095.

(31) Dlott, D. D. Optical phonon dynamics in molecular crystals. Annu. Rev. Phys. Chem. $1986,37,157-187$.

(32) Humphrey, W.; Dalke, A.; Schulten, K. VMD - Visual Molecular Dynamics. J. Molec.

Graphics 1996, 14, 33-38. 
(33) Alecu, I. M; Zheng, J. J.; Zhao, Y.; Truhlar, D. G. Computational thermochemistry: scale factor databases and scale factors for vibrational frequencies obtained from electronic model chemistries. J. Chem. Theory Comput. 2010, 6, 2872-2887.

(34) Neyertz, S.; Mathieu, D.; Khanniche, S.; Brown, D. An empirically optimized classical force-field for molecular simulations of 2,4,6-trinitrotoluen (TNT) and 2,4-dinitrotoluene (DNT). J. Phys. Chem. A 2012, 116, 8374-8381.

(35) Momma, K.; Izumi, F. VESTA 3 for three-dimensional visualization of crystal, volumetric and morphology data. J. Appl. Crystallogr. 2011, 44, 1272-1276.

(36) Dexheimer, S. L. Ed. Terahertz spectroscopy: Principles and applications. CRC Press, (2008).

(37) Joseph, J.; Jemmis, E. D. Red-, blue- or no-shift in hydrogen bonds: a unified explanation. J. Am. Chem. Soc. 2007, 129, 4620-4632.

(38) Dougherty, R. C. Temperature and pressure dependence of hydrogen bond strength: a perturbation molecular approach. J. Chem. Phys. 1998, 109, 7372-7378.

(39) Lutz E. T. G.; van der Maas J. H. Hydrogen bonds in crystalline carbohydrates: a variable temperature FT-IR study. J. Mol. Struct. 1994, 324, 123-132.

(40) Lutz E. T. G.; Veldhuizen Y. J. S.; van der Maas J. H.; Kanters J. A.; Baran J.; Ratajczak H. A variable low-temperature FT-IR study of crystalline beta-d-fructopyranose and deuterated analogs. J. Mol. Struct. 1992, 270, 381.

(41) Walther, M; Fischer B. M.; Jepsen P. U. Noncovalent intermolecular forces in polycrystalline and amorphous saccharides in the far infrared. Chem. Phys. 2003, 288, 261-268. 
(42) Moreno, D. V.; González, S. A.; Reyes, A. Turning symmetric an asymmetric hydrogen bond with the inclusion of nuclear quantum effects: the case of the [CN...H...NC] complex. J. Chem. Phys. 2011, 134, 024115.

(43) Afonin, A. V.; Ushakov I. A.; Vashchenko, A. V.; Kondrashov, E. V.; Rulev, A. Yu. GIAO, DFT, AIM and NBO analysis of the $\mathrm{N}-\mathrm{H} \cdots \mathrm{O}$ intramolecular hydrogen-bond influence on the ${ }^{1} \mathrm{~J}(\mathrm{~N}, \mathrm{H})$ coupling constant in push-pull diaminoenones. Magn. Reson. Chem. 2010, 48, 661670. 